\title{
Cultura de colaboración en un centro educativo: aportaciones desde el asesoramiento
}

\section{Collaborative Culture in a School: Contributions from Counseling}

\author{
Carlos Monge López ${ }^{1 *}$ \\ carlos.monge@uah.es \\ Jesús Domingo Segovia** \\ jdomingo@ugr.es \\ Juan Carlos Torrego Seijo* \\ juancarlos.torrego@uah.es \\ * Universidad de Alcalá, España \\ ** Universidad de Granada, España
}

\section{Resumen:}

Los procesos de cambio orientados a la mejora de la inclusión escolar suponen un elemento necesario en los centros educativos. En este sentido, el aprendizaje cooperativo se manifiesta en algunas instituciones educativas como una verdadera innovación que responde a esta temática. Para ello pueden utilizarse estrategias de asesoramiento colaborativo, considerando que la cooperación del alumnado ha de ir vinculada a la colaboración del profesorado y con otros agentes de la comunidad escolar. Una de estas estrategias viene definida por una serie adaptable de fases: (a) creación de condiciones para abordar la implantación del proyecto cooperativo y evaluación inicial de la red de enseñanza; (b) planificación y preparación para el desarrollo; (c) desarrollo y seguimiento del plan; (d) evaluación y propuestas de mejora. El objetivo principal de esta investigación es identificar y comprender las implicaciones esenciales de esta pro-

\begin{abstract}
:
Change processes focused on improving educational inclusion are a necessary element for schools. In this sense, cooperative learning appears to be a true innovation for educational institutions, giving a valid response for this issue. In order to pursue this goal, collaborative counseling strategies can be used, considering that students' cooperation must be aligned with teachers' cooperation and with the other agents involved in educational communities. One of these strategies is defined according to a number of adaptable phases: (a) creating the conditions in order to face the implementation of the cooperative project and initial assessment of the teaching network; (b) planning and implementation; (c) developing and monitoring the plan; (d) assessing and proposing improvements. The main goal of this research was to identify and understand the essential implications of this training proposal. To this end, we undertook a case study in a school of the
\end{abstract}

1 Dirección para correspondencia (correspondence address):

Carlos Monge López. Dpto. de Ciencias de la Educación. Universidad de Alcalá. Facultad de Educación. C/ Madrid, 1. 19001 Guadalajara (España). 
puesta formativa. Para ello se desarrolla un estudio de caso en un centro educativo de la Comunidad de Madrid, recabando la información a partir de entrevistas, grupos de discusión, análisis documental, observación y cuestionarios. Los resultados destacan el impacto en tres grandes ámbitos: en la organización escolar, en el desarrollo profesional del profesorado y en los aprendizajes del alumnado.

\section{Palabras clave:}

Aprendizaje cooperativo; asesoramiento colaborativo; desarrollo docente; estudio de caso; organización escolar.
Community of Madrid, collecting information extracted in interviews, focus groups, reviewing documents, observing and using surveys. Results highlighted an impact on three main areas: school organization, teachers' professional development and students' learning.

\section{Key words:}

Case study; collaborative counseling; cooperative learning; school organization; teacher development.

\section{Résumé:}

Les processus de changement visant à améliorer l'inclusion scolaire constituent un élément nécessaire dans les centres éducatifs. En ce sens, I'apprentissage coopératif se manifeste dans certaines institutions éducatives comme une véritable innovation qui répond à ce thème. À cette fin peuvent être utilisées des stratégies de conseil collaboratif, alors que la coopération des élèves doit être liée à la collaboration des enseignants et à d'autres acteurs de la communauté scolaire. L'une de ces stratégies est définie par une série adaptative de phases: (a) création de conditions pour aborder la mise en œuvre du projet de coopération et de l'évaluation initiale du réseau de l'enseignement; (b) planification et préparation pour le développement; (c) développement et suivi du plan; (d) évaluation et propositions d'amélioration. L'objectif principal de cette recherche est d'identifier et de comprendre les implications essentielles de cette proposition de formation. Pour cela, une étude de cas a été développée dans un établissement scolaire de la Communauté de Madrid, rassemblant des informations à partir des entrevues, de groupes de discussion, d'observation et de questionnaires. Les résultats mettent en évidence l'impact sur trois grands domaines: I'organisation scolaire, le développement professionnel des enseignants et l'apprentissage des élèves.

\section{Mots-clés:}

Apprentissage coopératif; conseil collaboratif; développement des enseignants; étude de cas; organisation scolaire.

Fecha de recepción: 7-2-2018

Fecha de aceptación: 21-5-2018

\section{Introducción}

La valía del aprendizaje cooperativo como metodología que involucra al estudiante y produce mejoras tanto en los resultados escolares como en la convivencia e inclusión está suficientemente estudiada y consolidada (Johnson y Johnson, 2014; Kadry y Safieddine, 2016; Laguador, 2014; Smith, Sheppard, Johnson y Johnson, 2005; Torrego y Negro, 2012). 
También se acumula un cuerpo importante de evidencias relevantes que hablan de la colaboración y la participación auténtica como condiciones imprescindibles para la inclusión educativa (Choi, 2011; FergusonPatrick y Jolliffe, 2018; Pujolàs, 2012) y para el despliegue de una ética democrática (Gozálvez, Traver y García, 2011). Así, no resulta extraño que un centro educativo apostase por desarrollar este tipo de metodologías. Ahora bien, resultaría anacrónico caminar hacia metodologías de aprendizaje cooperativo con un profesorado que trabajase de forma aislada, como es lo común en nuestro contexto (Bolívar y Bolívar, 2016) o con procesos formativos descontextualizados de su práctica profesional.

Cambiar el aprendizaje de los estudiantes supone también cambiar al profesorado. Puede resultar pretensioso intentar cambiar los resultados escolares, utilizando metodologías activas de aprendizaje en los estudiantes sin tomar en consideración el necesario incremento de la competencia profesional del profesorado en su contexto para operar dicho cambio. Sin esta mejora en el profesorado (tanto en formación como en generación de dinámicas y estructuras de soporte), los cambios serían meramente superficiales y poco sostenibles en el tiempo. Es bien conocida la máxima del techo de cristal de la mejora educativa que está en la capacidad profesional del profesorado. Pero se pueden mejorar las condiciones de la escuela para el desarrollo profesional de los docentes desde la mejora de los procesos de enseñanza-aprendizaje que se dan en el aula (Elmore, 2010). En este sentido, sería pertinente estimular y posibilitar procesos de reculturización del centro y de desarrollo profesional docente desde el empleo de la colaboración, la implicación y el empleo de metodologías como el aprendizaje cooperativo (Krecic y Grmek, 2008; López y Viáfara, 2007), para lo que podría ser un elemento central la acción del liderazgo educativo y compartido (Bolívar, 2014; Harris, 2014) también desde la función de asesoría (Torrego, Monge y Muñoz, 2018).

Desde que Escudero desde principio de los 90 fuese introduciendo en nuestro contexto algunos conceptos claves sobre la formación del profesorado, el centro con texto y contexto de formación, la mejora escolar y cómo apoyar estos procesos desde modelos de asesoría colaborativa, se ha venido acumulando un cuerpo de conocimiento y experiencia desde la Asociación para el Desarrollo y Mejora de la Escuela que dan soporte a iniciativas de innovación desde una filosofía educativa y un modelo versátil y flexible de asesoría heredero de tales aportes (Bolívar, 2012; 
Domingo, 2009; Escudero, 2009). Éste, siendo adaptado y consensuado con contextos particulares, se ha mostrado como una herramienta eficaz para dar soporte a procesos internos de mejora, como el analizado en este estudio de caso.

En este sentido, el estudio muestra los resultados de un proceso de asesoría que buscaba, a petición del centro educativo, facilitar que se operase el cambio en la institución, transitando hacia el aprendizaje cooperativo, con un asesoramiento también colaborativo al tiempo que fuese dejando huella, pistas de acción y experiencias exitosas que fuesen germen de un incipiente proceso de constitución como comunidad profesional de aprendizaje.

Con todo ello, el objetivo principal del trabajo consiste en identificar el impacto de una propuesta de asesoramiento colaborativo para la implementación de un modelo de aprendizaje cooperativo en el aula en un caso concreto. Dicho impacto se mide más allá del uso en las aulas de metodologías de carácter cooperativo, en términos de resultados educativos y de inclusión, además de la mejora en el desarrollo profesional del profesorado y de la cultura de colaboración en la institución.

\section{Marco teórico}

\section{La colaboración como elemento de mejora escolar}

La sociedad del siglo XXI presenta grandes desafíos que han de afrontarse desde la educación, y muchos de ellos exigen directamente respuestas desde un marco de colaboración e interdependencia (Johnson y Johnson, 2014). En este marco, la constitución de escenarios de relación y trabajo conjunto produce más que una simple suma de miradas o voces: juntos no sólo somos más, sino que colaborando somos mejores.

Tradicionalmente, la escuela es un escenario poco proclive para la colaboración, lo que la hace poco sensible hacia el cambio de creencias y prácticas pedagógicas. Ahora bien, también es la principal unidad básica de cambio. Hopkins, Harris, Stoll y Mackay (2010) señalan que la innovación plantea permanentes desafíos que cuestionan la práctica profesional y las dinámicas establecidas, lo que requiere posibilitar condiciones de diálogo profesional, de interdependencia positiva, de incremento de la responsabilidad individual, de interacción promotora, 
de desarrollo de habilidades sociales y de procesamiento grupal, con las que se van sustentando aquellos procesos de desarrollo profesional docente (Charner-Laird, Szczesiul, Kirkpatrick, Gordon y Watson, 2016; Johnson y Johnson, 2017). La transformación de estos espacios y culturas institucionales y profesionales hacia otras más colaborativas es un objetivo pertinente e imprescindible en la actualidad.

Hoy en día está bastante consolidada la idea de que la construcción de capacidades institucionales y profesionales viene dada por el avance de la colaboración profesional, el incremento del capital social y el desarrollo de comunidades de práctica profesional (Escudero, 2011). El conocimiento pedagógico acumulado señala que estas iniciativas deben estar enraizadas en su contexto (centro) y en su práctica profesional, contando con la promoción de tiempos, espacios y tareas que propicien la interacción profesional, el apoyo mutuo y la construcción conjunta de un proyecto/propósito de mejora. Ambos cambios debe operarse paralelamente, de manera que se apoyen mutuamente, lo que implica cambios culturales, organizativos y funcionales y lo que permite el avance hacia el desarrollo de comunidades profesionales de aprendizaje acompañadas de cerca por colegas críticos (asesoría colaborativa y liderazgo pedagógico y distribuido) que hagan posible y consistente este cambio (Escudero, 2011; Harris, 2011, 2014; Puroway, 2016).

Pero la colaboración no se instaura en el vacío, ni tan siquiera lo hace en una capacitación profesional, sino a partir de poner en valor el núcleo pedagógico del cambio y la colaboración (Elmore, 2010). Poner el foco en las estrategias de enseñanza (y que éstas vayan incrementado resultados educativos) dota de sentido a la mejora de la práctica docente. Mantener este foco y abordarlo desde la reflexión colectiva sobre las evidencias obtenidas, la interacción y el intercambio de las buenas prácticas es una nueva tarea de los líderes (Marsh y Farrell, 2015) que genera estructuras espacio-temporales y tareas comunes que desembocan en la configuración de capacidades profesionales, lo que se amplía y fortalece con el establecimiento de redes y alianzas entre centros y con otros profesionales críticos que amplíen el capital social y profesional de la institución.

De este modo, el incremento de este capital profesional de los centros se ha revelado como un elemento central desde el que estimular y posibilitar procesos de reculturización del centro (Hargreaves y Fullan, 2014), con el consecuente incremento de la capacidad organizativa de la escuela y del desarrollo profesional docente en una triple esfera (per- 
sonal, interpersonal y organizacional, siendo las colectivas las más relevantes). Como señala Harris (2011), aumentar el capital social de una escuela es el mejor modo para incrementar su capital profesional. La creación de capacidades profesionales requiere una responsabilidad colectiva donde los profesionales trabajen juntos para mejorar la práctica.

Stringer (2013) señala que esta construcción de capacidades es difícil de conceptualizar y generalizar, pues depende del tiempo y del contexto. No se trata sólo de que se cambie, sino de que éste aporte valor añadido a la práctica educativa, implique al profesorado y se sostenga en impulso a lo largo del tiempo. Va más allá de una mercadotecnia del cambio y del uso de unas determinadas pautas de acción, estando generalmente importadas de fuera de la escuela, sin llegar a impregnar la vida cotidiana ni la conciencia práctica profesional. La competencia para afrontar mejoras productivamente no siempre se produce adecuadamente $\mathrm{o}$ muestra fragilidad. Incluso muchas escuelas no cuentan previamente con dispositivos internos para su autorrevisión, por lo que suelen necesitar apoyo personalizado e incardinado en su proceso de desarrollo.

El estudio sobre experiencias profesionales de diseño, desarrollo y análisis de resultados de forma colaborativa (collaborative design) va acumulando progresivamente evidencias de que estos escenarios son una potente herramienta de desarrollo profesional (Svendsen, 2017; Voogt y otros, 2015). Esto implica también un liderazgo pedagógico y compartido del que puede formar parte el asesor. En este sentido, Escudero, Cutanda y Trillo (2017), recogiendo las palabras de una asesora, señalan que "es fundamental que alguien, además del equipo técnico (asesoramiento), tire del carro desde dentro, pues al fin y al cabo es el Claustro el que tiene que decidir si quiere o no formación" (p. 96).

\section{Asesoramiento desde una visión estrategia de procesos}

Desde esta perspectiva de puesta en valor de la colaboración profesional, como viene defendiendo Escudero (2009), la práctica educativa puede ser el "texto y contexto de formación". Es texto en cuanto a contenido básico y temática sobre la que desarrollar los procesos formativos en ejercicio, y es contexto como lugar, plataforma y escenario adecuado para poner en juego todos los procesos de reflexión, interrelación e intercambio de pareceres, experiencias y conocimiento pedagógico (práctico y teórico). 
La mejora de la educación necesita ir avanzando hacia comunidades profesionales comprometidas con el aprendizaje que superen las barreras al cambio (Murphy, 2015). Ellas son un escenario privilegiado de desarrollo profesional, pero necesitan un liderazgo pedagógico, democrático y distribuido (Harris, 2004), así como acciones de apoyo mutuo y asesoría para que éstas se constituyan y avancen (Bolívar, 2012), además de redes de apoyo (Katz, Lorna, Earl \& Jaafar, 2009). Pero no todos los modelos de asesoría son aptos para ello. En esta línea, los apoyos a la escuela deben ir dirigidos a aumentar la competencia del profesorado (no a suplantarla), a facilitar el desarrollo de los centros (colaborando en la renovación de su cultura, valores, procesos y prácticas) y hacia el desarrollo de prácticas educativas inclusivas con potencial para el buen aprendizaje de todos y entre todos.

Ha cambiado la gramática del cambio y, consecuentemente con ello, también ha variado sustancialmente tanto la gramática (forma de entender) como la dramática (puesta en escena) del asesoramiento. Las funciones tradicionales de apoyo a la mejora, en cambio, parecen mostrar mayor grado de permanencia. Desde el proyecto IPSI, existe un acuerdo sobre qué funciones debe desempeñar un asesor, pero no son asépticas y pueden tener consecuencias bien distintas en función del uso que se las dé.

La cuestión del perfil, de las funciones y de las tareas concretas que correspondan al asesor parecen una cuestión secundaria y derivable del modelo marco en el que se definen los supuestos del asesoramiento, sus objetivos y los procesos que pretende activar y a través de los que procura llevarse a cabo en el seno del sistema educativo (Escudero y Moreno, 1992, p. 91).

La asesoría, entendida como proceso de ayuda a un profesor o profesora, es una práctica socialmente comprometida que asume toda la tradición de un modelo colaborativo de proceso (Escudero y Moreno, 1992), precisada y resituada en este nuevo marco que debiera caminar en un escenario de construcción de capacidades de mejora, que replantea la situación hacia una óptica de "comunidades de aprendizaje profesional" para que todos se impliquen en la reconstrucción de la práctica docente en un escenario de debate y colaboración (Ben-Ari y Enosh, 2013; Bolívar, 2012; Bolívar, Domingo, Escudero, González y García, 2007). Por ello resulta pertinente hablar también de un modelo "colaborativo" 
de asesoría (Escudero, 2009; Guerrero, 2011; Lago, Pujolàs y Naranjo, 2011; Lago y Onrubia, 2011; Moruno, Sánchez y Zariquiey, 2011a).

Dicho modelo de proceso viene definido por una serie adaptable de fases, cuyo fin principal no es tanto el logro de implantar la mejora particular acometida, sino la dotación del propio proceso como marco y herramienta de autorrevisión (Domingo, 2005). Dichas fases se presentan más adelante al referirnos al establecimiento de la red de enseñanza (Moruno y Torrego, 2011). En este proceso es fundamental la participación activa de todo el profesorado implicado, la colaboración profesional y la recopilación sistemática de información para su posterior uso como evidencia sobre la que reflexionar colectiva y regularmente. En este sentido, la técnica está al servicio del sentido y del propio proceso de desarrollo, sin encorsetarlo, como apoyo sólido pero amigable.

Ahora bien, no puede olvidarse que resultan fundamentales los matices emergentes del conocimiento emocional de las situaciones de asesoría y desarrollo profesional sostenibles (Domingo, 2009; Kelly y Cherkowski, 2017). Ellos, en gran medida, pueden determinar bastantes posibilidades de éxito de esta práctica profesional, minimizando elementos disruptivos o promoviendo puentes y lazos estratégicos y afectivos que coadyuvan a la comunicación y la interdependencia. En definitiva, junto a la ética y la técnica, en estas prácticas también debe caminar la emoción, el clima y el significado compartido (hablar un mismo lenguaje y compartir mínimamente unos propósitos, una mirada, etc.), lo que implica cercanía, interacción, corresponsabilidad, propósito común, etc.

Es especialmente clarificadora la propuesta de Montero y Sanz (2008) cuando resaltan el valor de las dinámicas de colaborar e innovar como pegamento y vitamina para de asesoría, en un conjunto de variables interrelacionales y culturales que habría que cuidar.

En esencia, podríamos hablar de dos grandes ejes o nudos en torno a los que tejer la labor asesora en un centro educativo: por un lado, está el conjunto de actividades y tareas relacionadas con la colaboración y la cooperación (lo que hemos dado en Ilamar "pegamento") y, por otro, la actitud positiva, creativa para generar y dinamizar situaciones de cambios para la mejora (lo que con el mismo sentido del humor llamamos "vitaminas"); con más razón, si pretendemos que esos cambios perduren, porque llegan a ser sostenibles en el tiempo (p. 12). 


\section{Una propuesta de asesoramiento colaborativo para la implementación del aprendizaje cooperativo}

Para implementar el aprendizaje cooperativo en los centros educativos existen varias propuestas de asesoramiento colaborativo, entre las que destaca especialmente la consolidada en torno al Proyecto de I+D Orientado a la Excelencia "Incidencia del aprendizaje cooperativo en la inclusión de alumnos con altas capacidades en la Comunidad de Madrid" (EDU2013-47696-P). Básicamente, se estructura en cuatro grandes ámbitos de actuación: la cultura de la cooperación, la red de aprendizaje, las unidades didácticas cooperativas y la red de enseñanza.

La cultura de la cooperación (Moruno y otros, 2011a) enfatiza la idea del aprendizaje cooperativo como metodología de diferenciación curricular, quedando especialmente fundamentada bajo las teorías de Vygotsky, Bruner, Ausubel y Gardner. Resumidamente, este primer ámbito de actuación consiste en redefinir el proceso de enseñanza-aprendizaje con el fin de dar un papel central a las interacciones cooperativas entre iguales. El objetivo fundamental es generalizar el uso de estrategias cooperativas dentro de las diferentes aulas participantes, concienciando a toda la comunidad educativa de su utilidad y ventajas. Esto se trabaja principalmente a través de campañas de sensibilización diferenciadas según colectivos: equipo directivo, equipo docente, alumnos y contexto familiar.

El segundo ámbito de actuación de la propuesta hace referencia a la red de aprendizaje (Moruno y otros, 2011b), en la que trabajar elementos, procedimientos y secuencias. Los elementos básicos del aprendizaje cooperativo propuestos en el programa son: agrupamientos heterogéneos, interdependencia positiva, responsabilidad individual, igualdad de oportunidades para el éxito, interacción promotora, procesamiento interindividual de la información, utilización de destrezas cooperativas y evaluación grupal. En cuanto a los procedimientos y secuenciación, tres son las fases adaptables a cada contexto particular de centro y aula con el objetivo de que se instauren: en la primera fase (1-2 semanas) se trabajan técnicas cooperativas informales; en la segunda fase (1-3 meses) se agrupa al alumnado en equipos heterogéneos de cuatro miembros con los que trabajar técnicas cooperativas informales y destrezas de funcionamiento, introduciendo roles; en la última fase (3 meses) los equipos 
trabajan técnicas cooperativas formales e informales, así como destrezas de formulación y fermentación, perfeccionando los roles.

Las unidades didácticas cooperativas (Torrego y Zariquiey, 2011) se diseñan en función del diagnóstico de las características del alumnado, del grupo-clase y del profesorado, lo que lleva a establecer un proyecto en torno a una temática concreta planteando una situación-problema específico, un producto y una adaptación para las diversidades del alumnado.

Finalmente, el último gran ámbito de actuación se refiere a la red de enseñanza que se estructura en (Moruno y Torrego, 2011): (a) creación de condiciones para poder abordar la implementación del proyecto cooperativo; (b) planificación y preparación para el desarrollo; (c) desarrollo y seguimiento del plan; (d) evaluación y propuestas de mejora.

Durante la creación de condiciones se clarifica conceptualmente el tema básico del proyecto (aprendizaje cooperativo), se acuerda la metodología de trabajo y se hace frente a posibles resistencias. Un primer momento implica la clarificación de la tarea y sensibilización a través de una campaña informativa, una formación básica y la creación de un grupo interno (constituido por el coordinador del proyecto en el centro, el equipo directivo y el Departamento de Orientación, con el fin de consensuar las funciones y compromisos de todos). Y un segundo elemento queda constituido por la evaluación inicial de la red de enseñanza, en la que auto-revisar la formación del equipo docente, el agrupamiento del alumnado y la planificación de la implementación.

La planificación y preparación para el desarrollo se articula en torno a la estructura del equipo colaborativo y la propia preparación para implementar el proyecto. Después de una formación previa en habilidades de escucha activa y emisión de mensajes positivos, la estructura del equipo colaborativo contempla una serie de elementos de cara a desarrollar el aprendizaje cooperativo en el centro: liderazgo distribuido, reuniones eficaces, tareas de coordinación, contenidos específicos de trabajo, organización del trabajo colaborativo, etc. De cara a la preparación para el desarrollo, la propuesta implica establecer un perfil de cambio (clarificación de aquello que compete al docente en la implementación de la mejora perseguida) y un perfil de uso del cambio (descripción de las concepciones y prácticas docentes).

El desarrollo y seguimiento del plan se secuencia en tres etapas básicas a seguir: 
- Elaboración y acuerdo de criterios de observación entre profesores en sus aulas.

- Organización de parejas de observación.

- Organización de sesiones de seguimiento, lo que incluye la descripción de experiencias, el análisis en función de los objetivos planteados, la extracción reflexiva de conclusiones y la formulación de implicaciones para la mejora.

Para concluir la red de enseñanza se procede con la evaluación y propuestas de mejora, lo que se focaliza en tres elementos fundamentales:

- Evaluación del cambio y de la mejora, que implica un proceso de recogida, análisis e interpretación de datos para obtener una visión de los logros conseguidos con el proyecto.

- Evaluación para el cambio, que se concreta en procedimientos de recogida y análisis de información para la toma de decisiones continua durante el desarrollo del proyecto.

- Evaluación como cambio y mejora, que entiende que todo el propio proceso es evaluativo en la medida que todas sus fases suponen un marco de evaluación constante desde y sobre la realidad.

\section{Marco empírico}

La metodología de investigación se plasmó en un estudio de caso de tipo Il integrado, que se caracteriza por tener un único contexto, un solo caso y múltiples miradas de análisis, lo que facilita el acceso al campo, la comparación de evidencias y el análisis simultáneo de varios componentes (Yin, 2014).

El contexto en el que se enmarcó el caso analizado es un centro educativo de titularidad privada situado en la ciudad de Madrid, cuya metodología didáctica se articuló en torno a tres pilares básicos: la educación en valores, las competencias claves para el siglo XXI y la vanguardia en el desarrollo cognitivo. Para ofrecer una idea de su tamaño y número de miembros, cabe destacar que oferta los dos ciclos de Educación Infantil, seis niveles de Educación Primaria, Educación Secundaria Obligatoria, Bachillerato (Ciencias y Tecnología, Humanidades, Ciencias Sociales y Artes) y diversas actividades extraescolares. En la etapa de Educación Primaria, este centro estaba participando en varios proyectos de innova- 
ción docente y en contratos con el grupo de investigación "Inclusión y Mejora Escolar: Convivencia y Aprendizaje Cooperativo", a través de los cuales se estaba desarrollando una propuesta de asesoramiento colaborativo para la implementación de metodologías cooperativas e inclusivas (Moruno y Torrego, 2011). Este hecho favoreció el acceso al campo y la reducción de sesgos, pues el profesorado tenía especial interés por participar en la experiencia y ya venían desarrollando acciones similares anteriormente. Además, la negociación se trabajó considerablemente como parte del modelo de procesos del asesoramiento, consensuando espacios, tiempos, estrategias, funciones, responsabilidades, etc.

El caso quedó delimitado por el objetivo de investigación ya señalado al inicio (identificar el impacto de una propuesta de asesoramiento colaborativo para la implementación de un modelo de aprendizaje cooperativo en el aula en un caso concreto) y la formulación una serie de preguntas que pretendían identificar el impacto de esta propuesta de asesoramiento colaborativo y externo para la implementación del aprendizaje cooperativo en el centro: ¿qué impacto tuvo la propuesta en la organización escolar?, ¿cómo influyó en el desarrollo profesional del profesorado? y ¿cómo esto se reflejó en los aprendizajes del alumnado? Para dar respuesta a estas preguntas se recurrió a una serie de estrategias principales de recogida de información:

- Entrevistas estructuradas al equipo asesor, a todo el profesorado de Educación Primaria, al equipo de orientación y a la jefatura de estudios del centro.

- Escalas de tipo Likert a todo el profesorado de Educación Primaria en torno a sus prácticas y concepciones docentes.

- Observación no participante de los procesos de asesoramiento y de los procesos de enseñanza-aprendizaje dentro de las aulas de Educación Primaria.

- Análisis documental de producciones del profesorado, diarios reflexivos de asesoramiento y documentos de centro (Proyecto Educativo y Unidades Didácticas cooperativas).

- Grupo de discusión con el equipo asesor y el director del proyecto.

Ese equipo asesor estaba formado por dos asesoras y un director, como coordinador del grupo de investigación e innovación de la propuesta. En el momento del estudio, las asesoras contaban con un perfil académicoprofesional bastante similar (maestras, psicopedagogas, formadoras en 
aprendizaje cooperativo y estudiantes de doctorado con tesis vinculadas a la implementación y evaluación de esta metodología).

Con todo ello se pretendió triangular las fuentes e instrumentos como procedimiento de validez de la investigación cualitativa, además de realizar una confirmación con los participantes mediante el grupo de discusión junto con un trabajo prolongado durante tres cursos académicos (Sandín, 2000).

El procedimiento inductivo del análisis temático (Braun y Clarke, 2006) se complementó con otro deductivo siguiendo la propuesta de categorías de análisis del programa formativo utilizada previamente (Torrego y otros, 2018):

- Mejoras en el centro: (a) mejora de la imagen de la escuela y (b) estrategias organizativas que favorecen la innovación.

- Mejoras en el profesorado: (a) cambio de prácticas y concepciones docentes y (b) bienestar docente.

- Mejoras en el alumnado: (a) rendimiento académico, (b) relaciones sociales, (c) inteligencia emocional, (d) motivación académica, (e) modelo de convivencia y (f) inclusión escolar (políticas, prácticas y culturas).

Toda la información recogida fue analizada con ayuda del software Atlas. ti 7. En todo momento se comunicó a los participantes la finalidad de la investigación, así como los principios de confidencialidad comprometidos. Por ello, los nombres propios que aparecen a lo largo del documento son ficticios para evitar identificar a los participantes.

\section{Resultados y discusión}

Los resultados obtenidos, coincidiendo globalmente con otros trabajos similares (Torrego, 2016), muestran impactos positivos en tres dimensiones claras de mejora: (1) la organización y la imagen institucional por la expansión de su proyecto colectivo; (2) el desarrollo profesional docente, tanto en su dimensión institucional y colegialidad como en la personal más relacionada con la práctica docente (León, Felipe, Iglesias y Latas, 2011; Torrego y otros, 2018); (3) en las condiciones de inclusión y mejora de resultados académicos del alumnado (Johnson y Johnson, 2014; Moruno y otros, 2011a; Pujolàs, 2012). 


\section{Impacto en la organización escolar}

Esta experiencia de asesoramiento ha producido cambios relevantes en la organización escolar. El hecho más significativo fue la inclusión de principios y valores educativos en el ideario del centro. De hecho, el estilo pedagógico recogido en el Proyecto Educativo señaló que este colegio procuraba renovar su metodología basada en criterios que buscaban la atención inclusiva de la diversidad desde un enfoque cooperativo, así como el reconocimiento y valor de la pluralidad, promoviendo la participación de la comunidad educativa desde la colaboración. Esta idea venía fraguándose a lo largo de todo el proceso de asesoramiento, mudando la cultura escolar, pero se materializó en la etapa de evaluación, propuestas de mejora e institucionalización de la innovación a través de su concreción en los documentos prescriptivos de centro.

Renovamos nuestra metodología basada en criterios que buscan la excelencia educativa, la atención a la diversidad, el rigor científico, el trabajo cooperativo, la autonomía responsable, la enseñanza de idiomas, la sintonía con los avances tecnológicos y medios didácticos más eficaces. Ayudamos, mediante la reflexión diaria, a percibir el mundo circundante con sentido crítico, para saber descubrir los valores que encierra esta sociedad plural [...]. Nos sentimos responsables del espíritu y buena marcha del centro, nos sentimos invitados a enriquecerla con nuestra aportación y colaboración (análisis documental, Proyecto Educativo, 4:3).

Pero esta institucionalización no consistió en una mera inclusión en los documentos institucionales del centro, sino que se percibió como un verdadero cambio de la cultura escolar que favoreció la mejora de algunas prácticas docentes, considerando que la transformación colaborativa de la cultura escolar debe dirigirse al compromiso con la redefinición de las prácticas docentes (Ben-Ari y Enosh, 2013; Bolívar, 2012; Bolívar y otros, 2007).

En mi opinión, es una suerte la que tenemos de tener este asesoramiento que nos hace evolucionar y enriquecernos mutuamente. Además, como ya he comentado, está creando un ambiente innovador que no existía, porque ahora con el asesoramiento tenemos la oportunidad de conversar, testar, etc. (entrevista, profesora, 10:11). 
Partiendo de las condiciones organizativas de este colegio, se instauraron unos espacios y tiempos concretos destinados exclusivamente a la formación colectiva del profesorado en torno a la metodología cooperativa. Básicamente, antes de que el alumnado comenzase su jornada escolar, consistió en utilizar una hora de la jornada laboral del profesorado para esta tarea específica que se venía utilizando para otras labores, de tal forma que todo el equipo docente se reunía y participaba simultáneamente en la misma formación. En este sentido, como señalan Marsh y Farrell (2015), las nuevas tareas del liderazgo desde el asesoramiento deben ir destinadas, entre otras cuestiones, a capacitar a la organización escolar de recursos espacio-temporales para la formación compartida del profesorado. De hecho, cuando se habla concretamente de implementar el aprendizaje cooperativo resulta fundamental dotar al profesorado de condiciones de diálogo profesional para el desarrollo docente (Charner-Laird y otros, 2016; Johnson y Johnson, 2017). Este cambio en la organización escolar contribuyó a crear una identidad y un compromiso grupal hacia el proyecto de centro, si bien es cierto que dificultó contextualizar el asesoramiento a las diferentes realidades de cada aula. Sin embargo, esto no quería decir que se dejase de trabajar de forma individual con el profesorado y las necesidades de cada grupoclase, permitiendo así una mayor adaptación a los diferentes contextos dentro de la organización escolar.

Hay un cambio sustancial cuando se tiene un espacio concreto donde podíamos trabajar durante un tiempo más o menos dilatado, tranquilos... No podemos personalizar tanto, motivarles más y poder sacar más de sus necesidades [...]. Esto tiene la parte positiva de que se tienen que escuchar y generar un proyecto común. Uno puede aprovechar ese contexto de asesoramiento para crear más proyecto de centro, lo que antes eran proyectos por aulas. Pero es verdad que dificulta ese ajuste fino [...]. Yo creo que un asesoramiento individualizado es imprescindible mantenerlo en paralelo a esto. La persona individual con su grupo particular no puede perderse. Eso es... Al final, el profesor está pensando en su clase, en el día siguiente, en dónde siento a Fulanito y qué le digo (grupo de discusión, asesor, 32:32).

Esta combinación de formación individual y colectiva vino condicionada por algunos elementos organizativos trabajados especialmente durante la fase de asesoramiento orientada a la creación de 
condiciones, cuando se establecieron reuniones con el equipo directivo para consensuar la metodología de asesoramiento a desarrollar, las funciones y expectativas de cada una de las partes y la constitución de un grupo de trabajo. Precisamente, este último aspecto supuso una condición organizativa de gran relevancia para la implantación del aprendizaje cooperativo, contando con personas concretas y comprometidas que, como dicen Escudero y otros (2017), sean capaces de "tirar del carro".

El equipo directivo se muestra muy interesado y conforme con la propuesta de asesoramiento, dinamizando la participación del profesorado y facilitando una hora concreta de formación. También se ha creado un grupo interno en el que se dota de responsabilidad y poder al coordinador del proyecto dentro del centro educativo (diario de campo, investigador, 3:1).

De igual forma, también se evidenció cierto cambio de la imagen de la escuela dentro de su entorno más próximo, entendiendo que la participación de esta institución en el proyecto cooperativo había mejorado las percepciones de otros colectivos de la comunidad educativa. Como se recoge en otras investigaciones al respecto, las familias y Administraciones valoran positivamente que el centro educativo se comprometa con un proceso de cambio orientado a la mejora de la atención a la diversidad en la propia institución escolar (Torrego, 2016; Torrego y otros, 2018).

Hablando privadamente con Rosa [(asesora)], dice que el colegio está aprovechando para darse más a conocer dentro de su ámbito de actuación. Ha cambiado su ideario de centro y ahora lo está difundiendo entre la comunidad educativa, lo que cree que está teniendo bastante éxito de cara a la matrícula del alumnado y a la participación de las familias en la vida escolar (diario de campo, investigador, 2:1).

En definitiva, esta propuesta de asesoramiento para la implementación del aprendizaje cooperativo ha influido sobre la organización escolar, impactando especialmente sobre cuatro ámbitos principales: el ideario del centro educativo, la distribución horaria del profesorado, el funcionamiento de un equipo de trabajo y la imagen de la escuela. De todos estos elementos organizativos, la modificación de los tiempos do- 
centes fue percibida como la cuestión que más influyó directamente sobre el desarrollo profesional del profesorado.

\section{Impacto en el desarrollo profesional docente}

Dos fueron los focos principales sobre los que se percibieron impactos en torno al desarrollo profesional del profesorado. Por un lado, se encontraron mejoras en las concepciones docentes para pasar después a un cambio de sus prácticas educativas en el aula. Ese componente emocional en algunos profesores mejoró rápidamente, mientras que en otros fue un proceso mucho más lento y complejo. No obstante, los matices emergentes del conocimiento emocional suponen un elemento que no se puede olvidar en el asesoramiento (Domingo, 2009; Kelly y Cherkowski, 2017).

Ha habido un gran cambio con Susana del primer al segundo año. Por inseguridad parecía no querer animarse con el aprendizaje cooperativo, pero este año ha mejorado notablemente. Tiene muchas ganas de trabajar y de ver resultados, lo que ya ha conseguido en poco tiempo (entrevista, asesor, 23:23).

Ha costado que Juan cambiase sus concepciones sobre el aprendizaje cooperativo. Desde el comienzo fue una persona persistente y no quería implicarse en el proyecto de innovación. No obstante, las reuniones individuales del asesor con él han contribuido a cambiar esas concepciones poco a poco, de tal manera que cada vez iba participando más en el proyecto y diseñando actividades cooperativas diferenciadas (diario de campo, investigador, 2:17).

El hecho de mejorar las concepciones del profesorado también resultó fundamental para cambiar las prácticas docentes durante la fase de evaluación y propuestas de mejora, especialmente con aquellos más vigilantes, lo que se vio especialmente favorecido por utilizar estrategias ligadas a la calidez y transparencia de la figura asesora.

Úrsula se define públicamente como una persona vigilante y que al principio se sentía fiscalizada por la presencia de la figura del asesor, pero dice que la transparencia que la asesora tenía con ella hizo que la percibiese como un recurso enriquecedor destinado al aprendizaje de la organización escolar, del profesorado y del alumnado (diario de campo, investigador, 6:3). 
Dentro del asesoramiento colaborativo, especialmente durante la fase de creación de condiciones, resultó sumamente importante identificar a aquellos profesores con actitudes negativas hacia el aprendizaje cooperativo y desmotivados para cambiar su metodología de enseñanza, llegando entonces a mejorar ese componente actitudinal del profesorado y utilizándolo como criterio para la creación de grupos de trabajo con profesores heterogéneos para vivenciar la metodología cooperativa. Como afirman Moruno y Torrego (2011), la creación de condiciones se orienta hacia la identificación y superación de dificultades organizativas y actitudinales.

Una idea clave es identificar inicialmente a aquellos profesores más abiertos al cambio y que se han propuesto ellos para participar en este tipo de experiencias para establecer rápidamente una relación de confianza con esas personas. También es clave identificar a los profesores más cerrados, por así decirlo, para cambiar sus actitudes y hacerlos partícipes de la experiencia. Pero a veces tienes que tener cuidado con no destacar demasiado a aquellos que tienen mucha motivación e intentar dar voz a los que quizá se quedan atrás y no participan. Entonces, tienes que buscar la manera de hacer grupos mixtos donde las parejas estén en la Zona de Desarrollo Próximo (grupo de discusión, asesor, 37:22).

También mejoró la competencia de planificación del profesorado. Durante la fase de planificación y preparación para el desarrollo dentro de la propuesta de asesoramiento, el equipo docente logró un cambio altamente importante que le dotó de capacidades reflexivas e imaginativas. Antes de comenzar la experiencia de innovación en el centro, el profesorado venía utilizando casi exclusivamente las guías didácticas de las editoriales como elementos de planificación didáctica. A lo largo del primer año de asesoramiento fueron adaptando esas pautas a las características de sus grupos-clases. Y en el segundo año de asesoramiento fueron capaces de diseñar completamente sus propias unidades didácticas cooperativas y diferenciadas. Este hecho resultó muy costoso en algunos casos, especialmente en aquellos docentes más abstractos, mientras que fue más rápido y sencillo con aquellos profesores con más características vinculadas a la experimentalidad, reflexividad, creatividad, imaginación y abstracción. Esto se puede deber a que para la planificación se requiere cierta visión de futuro que posibilite ima- 
ginar el desarrollo de actividades antes de haberse realizado (Torrego y Zariquiey, 2011).

\section{Impacto en el aprendizaje del alumnado}

Como ya apuntaban otros estudios (Moruno y otros, 2011a; Torrego y otros, 2018), un gran avance fue la mejora de la motivación de los estudiantes hacia las tareas escolares. En este caso, esta idea queda fuertemente vinculada al desarrollo y seguimiento de las unidades didácticas cooperativas y diferenciadas.

En el caso de $2^{\circ}$ implantan toda la unidad didáctica cooperativa que diseñaron y vamos observando los resultados. Los niños están cada vez más contentos y suelen hacer muchas actividades en las que se coordinan con sus compañeros de las distintas clases. Hay muy buenos resultados en general (análisis documental, diario reflexivo de asesoramiento, 13:1).

Esta propuesta de asesoramiento colaborativo ha generado cambios en la metodología dentro de las aulas hacia un enfoque inclusivo mediante los elementos principales del aprendizaje cooperativo, pues cabe recordar que esta metodología viene demostrando su efectividad como estrategia de inclusión en las aulas (Choi, 2011; Ferguson-Patrick y JoIliffe, 2018; Johnson y Johnson, 2014; Moruno y otros, 2011a, 2011b; Moruno y Torrego, 2011; Torrego, 2016; Torrego y otros, 2018; Torrego y Zariquiey, 2011). Básicamente, la mejora de las habilidades sociales y de la cohesión grupal ha venido apoyada por tres de esos elementos: agrupamientos heterogéneos, interdependencia positiva y establecimiento de roles.

Los grupos se encuentran bien estructurados, conocen sus objetivos de grupo y han mejorado las habilidades sociales. Se gestiona bien el ruido y el tiempo. Hay buena gestión de los roles. Se ha mejorado la identidad grupal y el uso de técnicas simples a diario. A pesar de encontrar muestras de competitividad en algunos momentos, el ambiente cooperativo ha mejorado en general en este nivel, así como el nivel de ruido. Notamos cierta mejora en las interacciones frente a frente que realizan los alumnos entre sí, recurriendo más a menudo a las habilidades de ayuda y comunicación. Es notable la mejoría en los aspectos básicos del aprendizaje cooperativo. De hecho, po- 
demos comprobar cómo, en general, el grupo-clase trabaja en mayor medida las habilidades de ayuda y diálogo, con el fin de conseguir un buen resultado final de forma cooperativa. A pesar de esto, hemos de continuar por este camino, que se nota que está beneficiando a toda la clase. Este nivel ha avanzado muy bien en la implantación del cooperativo como metodología más diaria del aula, estando todo ello facilitado por la buena actitud y motivación del profesorado y las habilidades de ayuda que tienen los grupos en general. Con la introducción de pequeñas herramientas y nuevas técnicas, la clase ha mejorado sus habilidades cooperativas significativamente, encontrándose preparada para el diseño de sesiones cooperativas más complejas (análisis documental, diario reflexivo de asesoramiento, 3:13).

Los profesores se mostraron muy satisfechos con las mejoras conseguidas en el alumnado, especialmente con aquellas vinculadas a la gestión del ruido y la disrupción. No obstante, señalaron una lista casi innumerable de los beneficios que había traído el aprendizaje cooperativo apoyado en esta propuesta concreta de asesoramiento colaborativo. Por resumir algunas ventajas de las más recurrentes, éstas se pueden agrupar en: mejoras en las relaciones interpersonales, desarrollo de la empatía, aprendizajes significativos, valoración de la diversidad como elemento enriquecedor, toma eficaz de decisiones, competencia lingüístico-comunicativa, compañerismo, gestión óptima de recursos (tiempos

y espacios), etc. Y otros de los beneficios menos citados se pueden sintetizar en: mejora de la autoestima, de la creatividad y del compromiso por las actividades académicas.

\section{Conclusiones}

Los resultados informan que esta propuesta de asesoramiento colaborativo para la implantación del aprendizaje cooperativo lleva a una consecución de beneficios en la organización escolar, en el desarrollo profesional docente y en los aprendizajes del alumnado, lo que coincide con diferentes estudios (Goodyear, 2017) y las propuestas de la Association for the Study of Cooperation in Education (Gilroy, 2017), Ilevando también a cambiar ciertos aspectos de esta propuesta formativa de innovación cooperativa.

La necesidad de cambio y de querer responder al reto de la exce- 
lencia educativa, como propósito común instaurado desde el liderazgo de un centro educativo, le lleva a solicitar asesoría para implementar cambios en metodologías que motiven y que fomenten el aprendizaje y la inclusión. En particular, se interesan por seguir un programa de desarrollo del aprendizaje cooperativo y se sienten comprometidos en seguir explorando y mejorando muchos más elementos que este pilotaje ha puesto sobre la mesa.

En línea con las experiencias de collaborative design (Svendsen, 2017; Voogt y otros, 2015), los resultados informan que este profesorado aprende a colaborar diseñando, promoviendo y valorando colectivamente situaciones para que sus estudiantes cooperen. La apuesta decidida de este centro educativo y de sus líderes por la implantación de este modelo de aprendizaje en clase, en el caso objeto de estudio, ha generado un incremento de resultados educativos en los estudiantes. Se han observado impactos directos en la mejora de las relaciones sociales, la convivencia escolar y la inclusión educativa (todo ello fruto de la promoción de la interacción), generando, de paso, mayor grado de motivación e implicación de todos los estudiantes, con una mejora de los resultados de aprendizaje. Esto también ha servido como motor de desarrollo profesional. Si el profesorado observa resultados de mejora, se implica en aplicar este cambio y en desarrollarlo sin escatimar esfuerzos, lo que ya es, de por sí, interesante. Además, esta experiencia abre ventanas a la interrelación, al intercambio de experiencias, a la solicitud de apoyo mutuo y a la participación en procesos de mejora acompañados de colegas críticos que les ayudan en la puesta en marcha y seguimiento de este programa de cambio.

El asesoramiento colaborativo, y en particular el empleado para la implementación del aprendizaje cooperativo, directamente focalizado en la mejora de la práctica diaria y en los procesos de tareas de clase, en consecuencia con el incremento de satisfacción profesional por la mejora de resultados educativos, ha sido valorado y se percibe como un factor clave que incide favorablemente en el desarrollo profesional del profesorado. Esto mismo es consistente con los trabajos de Krecic y Grmek (2008), López y Viáfara (2007), Moruno y otros (2011a) o Pujolàs y Lago (2011). En este sentido, tanto profesorado como los líderes y asesores consideran que la acción de asesoría implementada, más allá de aportar el recurso técnico, el interés por el mismo y la formación, les ha capacitado para desarrollarlo, pues ha generado paralelamente 
un debate y consenso y relaciones de confianza mutua, promoviendo oportunidades de trabajo colaborativo que son herederas de la observación de resultados y de la participación activa en la implementación de prácticas educativas.

Todo ello implica también un liderazgo pedagógico y compartido, del que puede formar parte el asesor (Domingo y Ritacco, 2015; Harris, 2014) a modo de "colega crítico" que acompaña los procesos reflexivos y de desarrollo profesional desde cerca (Charner-Laird y otros, 2016; Escudero y otros, 2017; Schifter, 2016).

No obstante, estas conclusiones han de ser pensadas con cautela considerando las debilidades metodológicas del estudio. Se trata de un colegio particular comprometido con la mejora. Además, si bien se han triangulado las fuentes de información, no se toma en cuenta la voz del alumnado ni de las familias como informantes claves del proceso. Por ello, futuras investigaciones han de recoger estas voces para poder tener una visión menos parcial del fenómeno analizado.

\section{Referencias bibliográficas}

Ben-Ari, A., y Enosh, G. (2013). Power Relations and Reciprocity: Dialectics of Knowledge Construction. Qualitative Health Research, 23(3), 422-429.

Bolívar, A. (2012). Assesoria para la melhoria da escola e do trabalho na sala de aula. En J. Machado y J. Matias (Orgs.), Melhorar os processsos e os resultados educativos. O que nos ensina a investigação. (p.159-190) Oporto: Editor Fundação Manuel Leão.

Bolívar, A. (2014). Construcción de capacidades de la escuela: liderazgo compartido y comunidades profesionales de aprendizaje. Una propuesta de investigación. International Journal of Educational Leadership and Management, 2(2), 147-175.

Bolívar, A., y Bolívar, R. (2016). Individualismo y comunidad profesional en los establecimientos escolares en España: limitaciones y posibilidades. Educar em Revista, 62, 181-198.

Bolívar, A., Domingo, J., Escudero, J.M., González, M.T., y García, R.J. (2007). El centro como contexto de formación. En AAVV, Asesoría pedagógica. Módulo I. Madrid: MEC/CENICE. http://www.ite.educacion.es/formacion/materiales/89/cd/m1/modulo1.pdf

Braun, V., y Clarke, V. (2006). Using Thematic Analysis in Psychology. Qualitative Research in Psychology, 3, 77-101.

Charner-Laird, M., Szczesiul, S., KirpatrickK, C.L., Gordon P., y Watson, D. (2016). From Collegial Support to Critical Dialogue: Including New Teachers' Voices in Collaborative Work. Professional Educator, 40(2), 1-17. 
Choi, J. (2011). Review of the Research Trends and Effects of Cooperative Learning for Students with Disabilities. Journal of Special Children Education, 13(3), 49-75.

Domingo, J. (2005). Las prácticas de asesoramiento a centros educativos. Revisión del modelo de proceso. Education Policy Análisis Archives, 13(17), disponible en http:// epaa.asu.edu/epaa/v13n17/v13n17.pdf

Domingo, J. (2009). Asesoría a la escuela para la mejora de la enseñanza y el aprendizaje. En AAVV, Desarrollo de la gestión educativa en México: situación y perspectivas (pp. 101-126). México DF: Universidad Pedagógica Nacional.

Domingo, J., Y Ritacco, M. (2015). Aporte del Departamento de Orientación al desarroIlo del liderazgo pedagógico: un estudio desde la opinión de directores de Institutos de Enseñanza Secundaria en Andalucía. Educar em Revista, 58, 199-218.

Elmore, R.F. (2010). Mejorando la escuela desde la sala de clases. Santiago de Chile: Fundación Chile.

Escudero, J.M. (2009). Formación en centros y comunidades docentes de aprendizaje: teoría y práctica. En M. de Puelles (Coord.), Profesión y vocación docente (pp. 89118). Madrid: Biblioteca Nueva.

Escudero, J.M. (2011). Los centros escolares como espacios de aprendizaje y de desarrollo profesional de los docentes. En M.T. González (Coord.), Innovaciones en el gobierno y la gestión de los centros escolares (p. 117-142) Madrid: Editorial Síntesis.

Escudero, J.M., Cutanda, M.T., y Trillo, F. (2017). Aprendizaje docente y desarrollo profesional del profesorado. Profesorado. Revista de Currículum y Formación del Profesorado, 21(3), 83-102.

Escudero, J.M., y Moreno, J.M. (1992). El asesoramiento a centros educativos. Estudio evaluativo de los Equipos Psicopedagógicos de la Comunidad de Madrid. Madrid: CECCAM.

Ferguson-Patrick, K., y Jolliffe, W. (2018). Cooperative Learning for Intercultural Classrooms: Case Studies for Inclusive Pedagogy. Londres: Routledge.

Gilroy, P. (2017). Cooperative Learning: Exploring Challenges, Crafting Innovations. Journal of Education for Teaching, 43(3), 273.

Goodyear, V.A. (2017). Sustained Professional Development on Cooperative Learning: Impact on Six Teachers' Practices and Students' Learning. Research Quarterly for Exercise and Sport, 88(1), 83-94.

Gozálvez, V., Traver, J.A., y García, R. (2011). El aprendizaje cooperativo desde una perspectiva ética. Estudios sobre Educación, 21, 191-197.

Guerrero, Q. (Coord.) (2011). ¿Alguien necesita ayuda? Asesoría para la gestión y la mejora educativa. México, DF: SM/IEA.

Hargreaves, A., y Fullan, M. (2014). Capital profesional: transformar la enseñanza en cada escuela. Madrid: Ediciones Morata.

Harris, A. (2004). Democratic Leadership for School Improvement in Challenging Contexts. En J.E. MacBeach y L. Moos (Eds.), Democratic Learning: The Challenge to School Effectiveness (pp. 201-214). Londres: Routledge.

Harris, A. (2011). System Improvement through Collective Capacity Building. Journal of Educational Administration, 49(6), 624-636. 
Cultura de colaboración en un centro educativo: aportaciones desde el asesoramiento Carlos Monge lópez, Jesús Domingo Segovia y Juan Carlos Torrego Seijo

Harris, A. (2014). Distributed Leadership Matters: Perspectives, Practicalities, and Potential. Thousand Oaks, CA.: Corwin.

Hopkins, D., Harris, A., Stoll, L., y Mackay, T. (2010). School and System Improvement: State of the Art Review (keynote presentation). 24th International Congress of School Effectiveness and School Improvement, disponible en: http://www.icsei.net/ icsei2011/State_of_the_art/State_of_the_art_Session_C.pdf

Johnson, D.W., y Johnson, R.T. (2014). Cooperative Learning in 21st Century. Anales de Psicologia, 30(3), 841-851.

Johnson, D.W., y Johnson, R.T. (2017). The Use of Cooperative Procedures in Teacher Education and Professional Development. Journal of Education for Teaching: International Research and Pedagogy, 43(3), 284-29.

Kadry, S., y Safieddine, F. (2016). Cooperative Active Learning Methodology in Mathematics. En L. Gómez, A. López y I. Candel (Eds.), 8th International Conference on Education and New Learning Technologies (pp. 4039-4045). Barcelona: IATED.

Katz, S., LornamM., Earl, L.M., y Jaafar, S.B. (2009). Building and Connecting Learning Communities: The Power of Networks for School Improvement. Thousand Oak, CA: Sage.

Kelly, J., y Cherkowski, S. (2017). Collaboration, Collegiality, and Collective Reflection: A Case Study of Professional Development for Teachers. Canadian Journal of Educational Administration and Policy, 169, 1-27.

Krecic, M.J., y Grmek, M.I. (2008). Cooperative Learning and Team Culture in Schools: Conditions for Teachers' Professional Development. Teaching and Teacher Education, 24(1), 59-68.

Laguador, J.M. (2014). Cooperative Learning Approach in an Outcomes-based Environment. International Journal of Social Sciences, Arts and Humanities, 2(2), 46-55.

Lago, J.R., y Onrubia, J. (2011). Asesoramiento psicopedagógico y mejora de la práctica educativa. Barcelona: Horsori.

Lago, J.R., Pujolàs, P., y Naranjo, M. (2011). Aprender cooperando para enseñar a cooperar: procesos de formación/asesoramiento para el desarrollo del Programa CA/ AC. Aula, 17, 89-106.

León, B., Felipe, E., Iglesias, D., y Latas, C. (2011). El aprendizaje cooperativo en la formación inicial del profesorado de Educación Secundaria. Revista de Educación, 354, 715-729.

López, M.E., y Viáfara, J.J. (2007). Looking at Cooperative Learning through the Eyes of Public Schools Teachers Participating in a Teacher Development Program. Profile Issues in Teachers' Professional Development, 8, 103-120.

Marsh, J.A., y Farrell, C.C. (2015). How Leaders Can Support Teachers with Datadriven Decision Making: A Framework for Understanding Capacity Building. Educational Management Administration \& Leadership, 43(2), 269-289.

Montero, L., y Sanz, M.D. (2008). Entre la realidad y el deseo: una visión del asesoramiento. Profesorado. Revista de Currículum y Formación del Profesorado, 12(1), disponible en: http://www.ugr.es/ recfpro/rev121COL3.pdf

Moruno, P., Sánchez, M., y Zariquiey, F. (2011a). La cultura de la cooperación. El aprendizaje cooperativo como herramienta de diferenciación curricular. En J.C. Torrego 
(Coord.), Alumnos con altas capacidades y aprendizaje cooperativo. Un modelo de respuesta educativa (pp. 167-197). Madrid: Fundación SM.

Moruno, P., Sánchez, M., y Zariquiey, F. (2011b). La red de aprendizaje. Elementos, procedimientos y secuencia. En J.C. Torrego (Coord.), Alumnos con altas capacidades y aprendizaje cooperativo. Un modelo de respuesta educativa (pp. 199-251). Madrid: Fundación SM.

Moruno, P., y Torrego, J.C. (2011). La red de enseñanza para la implantación del proyecto. En J.C. Torrego (Coord.), Alumnos con altas capacidades y aprendizaje cooperativo. Un modelo de respuesta educativa (pp. 253-290). Madrid: Fundación SM.

Murphy, J. (2015). Creating Communities of Professionalism: Addressing Cultural and Structural Barriers. Journal of Educational Administration, 53(2), 154-176.

Pujolàs, P. (2012). Aulas inclusivas y aprendizaje cooperativo. Educatio Siglo XXI, 30(1), 89-112.

Pujolàs, P., y Lago, J.R. (2011). El asesoramiento para la transformación de las aulas en escenarios cooperativos. En E. Martin y J. Onrubia (Coords): Orientación educativa y procesos de innovación y mejora de la enseñanza (pp. 121-142). Barcelona: Graó.

Puroway, A.W. (2016). Critical Advising: A Freirian-inspired Approach. NACADA Journal, 36(2), 4-10.

Sandin, M.P. (2000). Criterios de validez en la investigación cualitativa: de la objetividad a la solidaridad. Revista de Investigación Educativa, 18(1), 223-242.

Schifter, C.C. (2016). Personalizing Professional Development for Teachers. En M. Murphy, S. Redding y J.S. Twyman (Eds.), Handbook on Personalized Learning for States, Districts, and Schools (pp. 221-235). Charlotte: IAP.

Smith, K.A, Sheppard, S.D., Johnson, D.W., y Johnson, R.T. (2005). Pedagogies of Engagement: Classroom-Based Practices. Journal of Engineering Education, 94(1), 87-101.

Stringer, P. (2013). Capacity Building for School Improvement. Roterdam: Sense Publishers.

Svendsen, B. (2017). Teacher's Experience from Collaborative Design: Reported Impact on Professional Development. Education, 138(2), 115-134.

Torrego, J.C., y Negro, A. (Coords.) (2012). Aprendizaje cooperativo en las aulas. Fundamentos y recursos para su implantación. Madrid: Alianza Editorial.

Torrego, J.C. (2016). Incidencia del aprendizaje cooperativo en la inclusión de alumnos con altas capacidades. En T. Ramiro-Sánchez, M.T. Ramiro, y M.P. Bermúdez (Coords.), IV Congreso Internacional de Ciencias de la Educación y del Desarrollo (p. 94). Granada: Universidad de Granada.

Torrego, J.C., Monge, C., y Muñoz, Y. (2018). Elementos de análisis para innovar la formación del profesorado en aprendizaje cooperativo y altas capacidades. En C. Monge y P. Gómez (Eds.), Innovando la docencia desde la formación del profesorado. Propuestas y realidades (pp. 297-317). Madrid: Editorial Síntesis.

Torrego, J.C., y Zariquiey, F. (2011). Unidades didácticas de aprendizaje cooperativo para alumnos con altas capacidades. En J.C. Torrego (Coord.), Alumnos con altas capacidades y aprendizaje cooperativo. Un modelo de respuesta educativa (pp. 291-260). Madrid: Fundación SM.

Voogt, J., Laferrière, T., Breuleux, A., Itow, R.C., Hickey, D.T., y Mckenney, S. (2015). 
Collaborative Design as a Form of Professional Development. Instructional Science, 43(2), 259-282.

Yin, R.K. (2014). Case Study. Research Design and Methods (5 edición). Thousand Oaks, CA: Sage.

\section{Agradecimientos}

Esta investigación se enmarca dentro del contrato de investigación "Conocimiento del alumnado, altas capacidades y aprendizaje cooperativo: un enfoque inclusivo" (UAH-94/2015), así como dentro del Programa Propio del Vicerrectorado de Investigación y Transferencia de la Universidad de Alcalá. 